\title{
Adolescencia y embarazo. Aspectos perinatales y socioeconómicos. Hospital de Maternidad Rafael Calvo C. Cartagena-Colombia
}

\author{
Jaime A. Barrios Amaya*; Alvaro Ramos Olier**
}

\begin{abstract}
RESUMEN: La presente comunicación pretende contribuir a la planeación, ejecución y evaluación de programas de acción dirigidos a la población de las madres adolescentes.

Estudiamos durante 4 años las adolescentes admitidas en el Hospital de Maternidad Rafael Calvo C., y en el Instituto de Seguros Sociales. El inicio de la vida marital fue de 14 años en-promedio, lo que demostró una iniciación sexual precoz. La vida obstétrica fue en promedio de 16 años. El $91 \%$ fueron primigestantes. Hubo complicaciones en el $27 \%$ de las embarazadas, siendo las entidades patológicas más frecuentes: Enfermedad hipertensiva del embarazo, Ruptura prematura de membranas, Hipodinamias, Partos prematuros, Desproporción cefalo-pélvica. La mayoría tuvo parto vaginal normal, y el resto tuvo parto por cesárea o parto complicado. El peso de los recién nacidos estuvo entre 2.500 y 3.500 gramos, aunque fue menor en el $10 \%$ de los casos.
\end{abstract}

Las causas que indujeron a las adolescentes a embarazarse fueron: pobreza, bajo nivel educativo, maltrato de los padres o padrastros, engaño, promiscuidad, malas compañías, desconocimiento de métodos de anticoncepción y de la biología de la reproducción.

Las consecuencias del embarazo en la adolescencia fueron:

aborto provocado, mayor morbimortalidad de los hijos, vivienda inadecuada y en hacinamiento, bajo ingreso, empleo de bajo estatus, rechazo de los padres o familiares y abandono del marido.

La investigación que of recemos, debidamente evaluada, puede ser instrumento de orientación a los profesionales que participen en programas dirigidos a satisfacer las necesidades de la población adolescente.

PALABRAS CLAVES: Adolescencia, embarazo, aspectos sociales.

SUMMARY: This communication is mean to contribute to the planning, execution and evaluation of programs directed to adolescent mothers.

We studied during four years the adolescent mothers received at the Hospital de Maternidad Rafael Calvo C. and at the Instituto de Seguros Sociales.

These mothers started marital life at a mean age of 14 years, and their obstetrical life started at 16 years in the average. Ninety-one per cent were primigravidae.

There were complications in $27 \%$ of pregnancies, and the most frequent pathologies were: Hypertensive disease of pregnancy, Premature rupture of membranes, Hypodynamia, Premature delivery, Cephalo-pelvic disproportion.

Most of the adolescents had normal vaginal delivery; the remaining cases had cesarean section or complicated vaginal delivery. The weight of the newborns was between 2500 and 3500 grams, although in $10 \%$ of cases, it was less than 2500 grams.

The reasons for adolescent pregnancies were: poverty, low educational level, ill treatment of fathers or step-fathers, deceit, promiscuity, bad companies, lack of knowledge of methods of contraception and of biology of reproduction.

The consequences of pregnancy in adolescents were: provoked abortion, higher morbi-mortality of children, inadequate housing, low income, low-status employment, rejection of parents or relatives, abandon of husband.

This research, duly evaluated, can orientate professionals who work in programs directed to satisfy the needs of the adolescent population.

KEY WORDS: Adolescence, pregnancy, social aspects.

La adolescencia la entendemos como una etapa del desarrollo humano dentro de la cual se incorporan las estructuras de los períodos previos, y cuyas manifestaciones individuales dependen del medio cultural. No es posible decir con precisión cuándo empieza y cuándo termina, más para la ejecución del presente concepto la consideramos comprendida entre los 12 y los 17 años. \footnotetext{
* Profesor Titular. Departamento de Obstetricia y Ginecología. Facultad de Medicina Universidad de Cartagena. Ex-Jefe del Departamento de Obstetricia y
Ginecología. Universidad de Cartagena. Ex-Vicerector Académico. Universidad de Cartagena.

** Profesor Titular. Facultad de Medicina. Departamento de Obstetricia y Ginecología. Universidad de Cartagena.
} 
Durante la adolescencia, más que en cualquier otra época de la vida, diversos factores e impulsos de tipo biológico, psicológico, cultural y social, interactuando, influyen significativamente en la evolución de la sexualidad y el manejo que de ella hagan las jóvenes. Por lo tanto, para juzgar la normalidad del desarrollo sexual de las adolescentes, tenemos que conocer las principales circunstancias ambientales y personales de tipo cultural, económico, ético y biológico, que son las más importantes que modelan y alientan ese actuar.

En este período la sexualidad no puede ser considerada en forma aislada, por el contrario debe ser vista como íntimamente ligada e integrada con todo el proceso biosicosocial de esta etapa de vida y en base en lo acontecido durante la infancia y la niñez. Es necesario recordar que la sexualidad bien entendida implica lo que realizamos, pero también lo que somos. Es una identificación, una actividad, un proceso biológico y emocional, una perspectiva y una expresión de sí misma. Está fuertemente influida por las creencias sociales y personales, y a su vez influye fuertemente tanto en las creencias como en la conducta.

Durante el período de 1980 a 1988 (análisis retrospectivo) la Maternidad dio asistencia a 33.234 mujeres; de ellas, el $88 \%$ demandó servicios de parto, y el $12 \%$ asistencia relacionada con aborto.

Del total anterior se revisaron 18.559 Historias Clínicas (al azar) y dentro de este universo 2.070 correspondieron a menores de 18 años, cifra que equivale al $11 \%$ del total anotado. De ellas el $90 \%$ demandó servicios relacionados con el parto y $10 \%$ por aborto.

Dentro de la población total atendida entre 1980 y 1988 (33.234 pacientes) el 6\% corresponde a jóvenes por proceso de parto y el $5 \%$ acudió por problemas relacionados con aborto.

Entre el grupo de jóvenes cuyo expediente fue estudiado, se encontró una distribución de edades que otorga el primer lugar a los 17 años (57\%), segundo a los 16 años (30\%), tercero a los 15 años (10\%), el cuarto correspondió a los 14 años (2\%) y finalmente las edades de 13,12 y 11 años aportaron el quinto lugar con tan sólo un 1\%.

El análisis de la muestra retrospectiva reveló de manera contundente que las madres usuarias fueron en un $74 \%$ urbanas, dejando a las de origen rural un 22\%. En $4 \%$ de las Historias el dato no fue anotado.

Un estudio de las calificadas como urbanas puso de manifiesto que el $65 \%$ de ellas procedian de los barrios pauperizados y marginales de la ciudad; $32 \%$ eran residentes de zonas obreras y populares, y tan sólo $3 \%$ correspondió a repartos de clase media.

La extensa muestra consultada afirma que el $70 \%$ fueron madres solteras y $23 \%$ madres casadas. En un $7 \%$ sin dato.

La Historia Clínica mostró, entre otros datos, que la actividad sexual la iniciaron el 36\% a los 16 años, 25\% a los 15; $16 \%$ a los 17; $10 \%$ a los 14 años; el 3\% lo forman en suma aquéllas que se iniciaron entre los 13, 12 y 11 años. La misma fuente ofrece los datos de iniciación de la vida obstétrica.

Las adolescentes estudiadas en este grupo fueron en un $90 \%$ primíparas, $7 \%$ cursaban una segunda gestación, y el $3 \%$ eran grávidas por tercera vez.

En la investigación retrospectiva, de aquéllas que buscaron atención por aborto, el $74 \%$ registró este acto por primera ocasión, un $4 \%$ por segunda, y lamentablemente en el $22 \%$ este dato no fue anotado.

Entre estas pacientes, la mayoría (el 85\%) no recibieron atención prenatal privada o institucional, de manera que el síntoma que las trajo a nuestra institución fue el dolor del trabajo de parto; el sangrado por genitales externos fue síntoma en un 11\%, otros motivos de poca significación estadística en esta serie fueron convulsiones, retención de placenta, etc.

La mala información, la poca educación sexual, el subempleo y el desempleo son factores agravantes.

El estado civil cuenta entre los factores determinantes de la movilidad social de una población.

El material que nos ocupa revela de manera insoslayable que predomina en la población de madres adolescentes el estado civil de solteras, con un porcentaje de $81 \%$. Aquí quedan incluidas las uniones libres estables, uniones libres inestables, y quienes están separadas. El mayor contribuyente a este porcentaje está dado por un $56 \%$ que corresponde a solteras en unión libre inestable.

La unión libre inestable la entendemos como el abandono del compañero durante un lapso, para más tarde regresar, establecer nuevamente relaciones, dejando como producto de ellas casi siempre otro hijo, que será también abandonado junto con su madre. Esta forma de relación va convirtiéndose en una especie de juego con manifestaciones de inseguridad, inestabilidad e inmadurez, que llevan finalmente a la ruptura de relaciones entre la pareja y los lazos familiares, dejando ya irremisiblemente en situación de total desesperanza a la mayoría de las jóvenes que quedan solas para criar a sus hijos.

Las adolescentes separadas (9\%) pueden ser un indicador del arraigo de prejuicios sociales en nuestro medio en relación con el vínculo matrimonial, ya que estas jóvenes fueron presionadas a contraer nupcias. Los padres de la adolescente creen que el matrimonio es la solución del problema del embarazo precoz. En la mayoría de los casos el joven acepta casarse con la compañera bajo presión ejercida por los padres de ésta o de ambos, mas plenamente consciente y programado que luego de casado no vivirá más con la joven.

Las madres separadas o abandonadas no cuentan con el apoyo económico de su cónyuge ni de los familiares de él; los datos del estudio señalan una visión lamentable de la pareja dada su inestabilidad y en múltiples formas se manifiestan sentimientos de vergüenza, retraimiento, depresión, ausentismo de los grupos de su misma edad y rechazo por parte de los padres de sus amigos. 
Otros adolescentes se casan o conforman una familia en unión libre con plena conciencia. Sus motivaciones pueden ser neuróticas, mas asíbuscan un ambiente satisfactorio que el de su hogar de procedencia, donde no encuentran paz, comprensión, apoyo. Otros, por la presión y autoritarismo de que son víctimas; algunos para liberarse del mal trato físico y emocional, o bien lograr separarse de sus mayores tratando (aunque por la vía equivocada) de ser aceptados como hombres o mujeres.

Nivel de escolaridad. La educación es el medio principal con que cuenta el grupo social para afirmar la personalidad de los individuos enriqueciéndolos con la capacidad de afrontar las contingencias de la vida y crear oportunidades de superación. Es así mismo un instrumento para establecer las mejores relaciones recíprocas entre los miembros, velar por la conservación de los valores del patrimonio cultural, promover y afianzar en los individuos la adquisición de intereses que propendan a un mayor bienestar individual y colectivo. Por lo dicho se desprende la fundamental importancia que tiene la educación para la vida diaria, lo importante que es comenzarla temprano, y relacionarla con la realidad del medio ambiente y con las experiencias cotidianas.

Si el nivel de escolaridad no puede considerarse como un factor determinante de un embarazo, sí puede ser considerado como un factor condicionante, al favorecer una predisposición a la iniciación de una vida genital temprana.

Es así como el $44 \%$ cursó su primaria completa, y el 35\% primaria incompleta. De acuerdo a su edad cronológica, deberían estar cursando la educación media, más la realidad es que el $80 \%$ de la población estudiada no ha superado el nivel primario o elemental.

Tan sólo un $18 \%$ de las jóvenes han cursado algún grado de educación media, y sólo el $0.65 \%$ concluyó este nivel. Algunas han dejado sus estudios por causa de una relación sexual prematura o un embarazo precoz.

En la población investigada, la educación ha sido y es predominantemente no formal; es aquélla adquirida o aprendida por tradición oral y ejemplo de vida, comunicación existencial de padres a hijos, y no requiere de un plan de estudio establecido. Es importante considerar los aportes que la educación no formal ofrece a las personas, lo que ella por sí sola ha servido en el proceso de desarrollo de estos núcleos de población; de hecho ha conformado toda una subcultura denominada de la marginación, la cual está marcada por los mitos, tradiciones y tabúes. Estos, por muy aberrante que a nuestro concepto cultural parezca, son hitos en la conducta social y emocional de nuestras pacientes y habrán de tenerse en cuenta y manejarse con respeto en el diálogo y la convivencia de relación paciente-agente de la salud.

La educación formal, cuando es llevada a estos sectores, debe, a través de programas que retomen estas actitudes culturales, darles una orientación que las incorpore, legitimándolas en lo que de valioso y positivo tengan dentro del contexto cultural "formal" y haciéndolas funcionales en la comunidad.

Actividad ocupacional e ingresos. Analizando los resultados se puede observar que el $72 \%$ de la población estudiada se desempeña en actividades inestables que no les permiten un ingreso familiar constante, concretando sus aspiraciones hacia el sustento diario, limitándose las satisfacciones de otras necesidades básicas como la educación, salud y vivienda, que permitan alcanzar un equilibrio en el bienestar social.

Así, pueden calificarse como trabajadores independientes el 3\% (esta denominación implica que no tienen patrón); su trabajo consiste en vender comestibles en su casa o en el mercado público. El $7 \%$ de casos estudiados desarrollan actividades que corresponden a empleo en el cual se les brindan las respectivas prestaciones y derechos. Finalmente observamos que el $18 \%$ de pacientes no tienen oficio remunerativo alguno, son desempleadas habituales, más aún, nunca han trabajado.

Las actividades en que se ocupan estas madres adolescentes muestran la capacidad de ingreso y bienestar familiar. Teniendo en cuenta que el ingreso es un indicador del nivel de vida de la familia, ya que determina la capacidad de consumo y de éste depende la satisfacción de las necesidades básicas, podemos colegir que su condición de vida no va hacia un bienestar integral, sino más bien a un estancamiento determinado por el tipo de ingreso y actividad.

El nivel de ingresos es muy bajo y por tanto no les permite la satisfacción integral de las necesidades básicas. El $71 \%$ devengan un sueldo por debajo del salario mínimo. Las únicas que obtienen salario superior al mínimo en el universo estudiado son las que trabajan de manera independiente.

Los gastos son por lo general mayores que los ingresos. Esto demuestra claramente que estas jóvenes no están en capacidad y oportunidad de gozar de una alimentación adecuada, ni de obtener una mejor educación, y de mejorar las condiciones de su vivienda.

Situación familiar. La familia es el medio dentro del cual el niño y el adolescente reciben las normas, costumbres y valores de la sociedad. En nuestra cultura la familia es un grupo asociativo, basado en la libre elección del cónyuge y una sanción oficial (matrimonio religioso o civil). Todo lo que se sale de estos parámetros es rechazado por la sociedad.

El ambiente familiar y social en que se haya desarrollado el adolescente va a tener una influencia decisiva en su comportamiento. Existe en cada joven una única adolescencia, pero su personalidad está marcada por el medio donde se organizó. El agente de cambio, ambiente social, ejerce una acción más decisiva que la acción del ambiente familiar.

Generalmente el adolescente exige una independencia y una libertad mayor a la que sus padres le conceden. Para manejar esta situación emplea mecanismos de amenaza, como irse del hogar, contínuas rabias y protestas, maltrato a sus padres, etc. El objetivo es desconocer la autoridad y las normas establecidas, y expresar que ya no son las mismas que antes.

Frente a las sanciones responden con desafío, mentiras, o se valen de terceros (hermano o amigo) que disfracen la situación ante sus padres. 
Aunque la resistencia y el rechazo a la autoridad se ve en ambos sexos, los hombres suelen manifestar más su inconformidad. La mujer adopta otros mecanismos sutiles; malgenio continuo ollanto silencioso. No obstante, en ocasiones el rechazo a los padres lo realizan teniendo relaciones sexuales o casándose prematuramente. Las entrevistas con nuestras pacientes corroboran y matizan en abundantes ejemplos todo lo antes expresado.

Por otra parte, la falta de apoyo emocional de los familiares de las madres adolescentes es un hecho frecuente real, y sabiéndolo o no, los padres están influyendo negativamente en el porvenir y bienestar de la joven, ya que ellos creen que una vez la joven ha conseguido un compañero, cesan sus obligaciones como responsables tanto en lo emocional como en lo económico. La mayoría de los padres ignora que en esta edad todavía la joven está en proceso de formación, no habiendo alcanzado su plena madurez psicológica, y requiere de su ayuda para poder afrontar la situación que está viviendo. Nuestro estudio avala esta bpinión a la luz de entrevistas con jóvenes y sus familias.

Las Historias Clínicas integradas de estas jóvenes revelan que el 37\% de las pacientes tienen familiares que no les brindan ayuda económica y emocional.

El $33 \%$ corresponde a la familia colaboradora, lo que significa que la familia de la paciente se hace presente y responsable de la situación de la adolescente.

El 16\% corresponde a los casos de abandono, la adolescente se encuentra en completo desamparo por parte de padres y familiares.

En el $14 \%$ de los casos, la paciente cuenta parcialmente con la ayuda de su familia.

El nivel económico, social y cultural de los padres ejerce influencia decisiva en el comportamiento y actitudes de la joven frente a sí misma y a los demás.

Analizando las estadísticas antes mencionadas vemos que ante el embarazo precoz de una adolescente, los padres presentan según los rasgos particulares de su personalidad y las actitudes de comprensión ante el problema, toda una gama de reacciones, unas favorables y otras claramente perjudiciales a la solución del mismo. Algunos traducen un intento de negación al problema ante un oscuro sentimiento de culpa, otros aceptan la realidad con frío pesimismo, pero hay algunos, afortunadamente, que conociendo la realidad se proponen una tarea constructiva.

Tamaño del grupo familiar. Las madres solteras embarazadas provenían en un $42 \%$ de grupos familiares compuestos entre 4 y 6 miembros; un $31 \%$ de núcleos familiares de 10 y más miembros; el $21 \%$ de familias formadas entre 7 y 9 miembros, y un $6 \%$ de hogares constituidos por 2 a 3 miembros.

Sabido es que a mayor grupo familiar, menores serán las posibilidades para un buen desarrollo físico, educativo, emocional, con cariño, comprensión y adecuada comunicación, dado que el número de hijos no permite la individualización de las atenciones que prestan los padres.

Relacionado el número de familiares con el nivel de ingreso y distribución de la vivienda se puede observar que hay un alto índice de hacinamiento, lo que trae como consecuencia precarias condiciones de salubridad, comportamiento social inadecuado (vocabulario y manifestaciones grotescas, imitación de comportamientos inadecuados de los adultos). El factor hacinamiento es determinante en el despertar a edad muy temprana del instinto sexual, lo que lleva a iniciar precozmente esta relación, y si a ello se agrega la falta de una buena educación sexual, generada en la mayoría de los casos por el bajo nivel de escolaridad familiar y los aspectos culturales imperantes, además de la vivencia directa de los hijos de la conducta genital de sus padres, podremos entender mejor la conducta de los y las jóvenes en su relación genital.

La tenencia y estado de la vivienda. El $61 \%$ de las entrevistadas vive en la que denominamos "alojada invadida", lo que significa que es una morada construida con materiales desechables en terrenos que no les pertenecen, sino que han tomado por la fuerza; también engloba a aquellas jóvenes que viven esporádicamente alojadas en casa de un pariente o amigo. El $17 \%$ corresponde a las que viven en sus casas propias; $13 \%$ vive en casa arrendada, pudiendo ser tan sólo una pieza o apartamento compartido. Finalmente, el grupo denominado "amortizada", que es un 6\%, significa que la vivienda le ha sido adjudicada a través de un instituto o corporación de la vivienda.

La casa habitación. Al igual que la tenencia de la vivienda, la estructura y distribución de la misma es también un problema, ya que no cuentan con los requisitos mínimos, como ventilación, luz, privacidad, servicios sanitarios, etc., que les proporcionen una forma de vivir cómoda y digna. La distribución y construcción de la vivienda es variada.

El 34\% reside en una mala choza, construida en material de desecho y poca seguridad, carece de servicios públicos y de infraestructura, cuenta con una o dos piezas en donde realizan actividades como dormir, cocinar, comer, descansar, etc. Es frecuente encontrar en este tipo de vivienda de 4 a 7 personas, lo que significa compartir muchas actividades en tan limitado espacio. Las consecuencias de esta situación se reflejan en la salud y el comportamiento social de las personas, porque marca los patrones de conducta, por ejemplo: se mantiene la dependencia, hay promiscuidad, hacinamiento, etc.

El $61 \%$ viven en una habitación en regulares condiciones, construida en material de bloque y ladrillos, cocina y servicios sanitarios; cuenta con servicios públicos y alguna obra de infraestructura.

El $5 \%$ vive en una habitación buena, construida con materiales de buena calidad; la distribución de la vivienda responde a las necesidades de habitación de la clase media. Cuenta con todos los servicios de infraestructura. Este porcentaje es bajo en relación con los demás, y con esto se demuestra que la mayoría de las entrevistadas pertenecen a la clase menos favorecida. 
También es importante mencionar el aspecto relacionado con el tratamiento de los desechos, entre ellos las basuras y excrementos. Es costumbre habitual de estas familias arrojar las basuras y excretos al patio o solares, hábito que contribuye a la proliferación de diversas enfermedades.

La carencia de vivienda, de servicios sanitarios u otros medios de sanidad, sumada al poco conocimiento sobre saneamiento ambiental, influye en la mala utilización y tratamiento que a éstos se les da, y se convierte en una costumbre general debido a que en los barrios donde viven estas madres adolescentes es escaso o nulo el servicio de recolección de basuras, agua potable, alcantarillado y otros.

El parto vaginal normal fue el más frecuente, así lo manifiesta la cifra de 89\%; la cesárea ocupa el segundo puesto, con un 6\%, en tanto que los partos conducidos suman el 3\%, y los partos inducidos el $2 \%$.

Los niños producto de la gestación en estas jóvenes madres fueron normales en un $90 \%$, calificando sus condiciones neonatales. Por su peso, la gran mayoría puede calificarse de igual manera, ya que el $69 \%$ lograron un peso entre los 2.500 y 3.500 grs. Los productos calificados como con peso bajo al nacimiento sólo fueron el $10 \%$. Niños con un peso superior al de 3.500 grs, registraron el $15 \%$.

Ciertos estudios mencionan que algunas complicaciones importantes durante el embarazo, como toxemia, anemia, prematurez, parto prolongado y complicaciones post-parto, aumentan en esta etapa de la vida. Otros estudios recientes demuestran que cuando se proporciona una amplia atención obstétrica disminuyen las complicaciones en este campo.

Nuestra experiencia demuestra que en la población adolescente atendida en nuestro medio, sólo un $27 \%$ presentó patología obstétrica. Entre las más frecuentes se registraron: enfermedad hipertensiva del embarazo, 26\%; ruptura prematura de las membranas, $21 \%$; hipodinamia, $10 \%$; parto prematuro, $9 \%$; desproporción cefalopélvica, $9 \%$; diversas presentaciones anómalas, $7 \%$; amnionitis, $6 \%$; retención placentaria, 3\%; endometritis puerperal, 3\%; infección urinaria, 3\%; para las entidades nosológicas placenta previa, desprendimiento prematuro de la placenta normoinserta, y mola hidatiforme, se tuvo al $1 \%$ para cada una. Se presentaron otros grupos menos significativos de alteraciones, que entre todos sumaron un $1 \%$.

Las dos preocupaciones médicas que surgen por la conducta sexual de las adolescentes son con mayor frecuencia el embarazo y las enfermedades venéreas.

¿Cómo solucionar estos problemas? Las posibilidades para actuar como consejero sexual son muy limitadas. Las adolescentes son renuentes a hablar de su vida sexual dentro de una relación convencional médico-enferma, consideran al facultativo una prolongación de sus progenitores. Con empeño puede llegarse a muchos adolescentes y conocer sus preocupaciones, pero es menester conocer a fondo y precisar cuáles son estas preocupaciones a las que se enfrentan.

Si la ignorancia, promiscuidad, ausencia de educación, dirección y apoyo pueden ser en las marginadas una causa predisponente y hasta condicionante de su embarazo, para las adolescentes de clase media, el embarazo es a menudo resultado de intentos frustrados por resolver los conflictos psicológicos específicos de su desarrollo. Cuando la patología psicológica o social grave es la causa del embarazo, el fenómeno se acompaña de otros patrones de mala adaptación, como uso y abuso de drogas, delincuencia, abandono de la familia o fuga de la escuela, causas todas ellas que pueden también señalarse entre las marginadas.

La ignorancia sobre las funciones sexuales y las supersticiones o temores relacionados con el uso de los anticonceptivos contribuyen también al problema del embarazo no deseado.

Una proporción pequeña de adolescentes desea el embarazo, y un número probablemente igual es indiferente ante la posibilidad del embarazo después del coito.

Para algunas de estas jóvenes el embarazo puede representar un intento constructivo de afrontar los problemas inherentes a la adolescencia. Por desgracia es difícil que estas mujeres lleven a cabo más tarde un matrimonio estable, o que creen un ambiente saludable para el recién nacido, aunque algunas madres jóvenes logran triunfar. Los factores importantes en las que logran el triunfo son:

1. Existencia de una persona responsable que proporciona los medios de seguridad emocional y económica necesarios hasta que la adolescente se puede bastar por sí misma.

2. Concepto realista del papel materno.

3. Motivación de que el embarazo ha sido por razones positivas y no por razones autodestructivas o neuróticas. Es útil valorar los factores personales, familiares y sociales que influyen en la adolescente embarazada, para que el médico se oriente hacia alternativas disponibles para atender el embarazo y la posibilidad de planear otros embarazos futuros.

Para la adolescente urbana, el embarazo se complica más por pobreza, desajuste social, y falta de comunicación. Los resultados por lo tanto dependen de que se conozcan los diversos factores etiológicos que intervienen, las necesidades específicas de la adolescente y la disponibilidad de diversos recursos comunitarios.

Medidas sanitarias preventivas. Las adolescentes embarazadas pueden ser consideradas como un grupo de riesgo de repetición del embarazo, causando problemas sociales, educacionales y sanitarios futuros. Los niños productos de los embarazos de la adolescencia corren peligro de gran variedad de problemas, como abandono y desarrollo físico y emocional retrasado. 
El éxito en la mayoría de las pacientes adolescentes depende en gran parte de los buenos servicios obstétricos, psicológicos, sociales y educacionales.

La educación durante el embarazo, que comprende educación sexual, preparación emocional y actitud positiva hacia el embarazo, planeación familiar, nutrición, efectos de fármacos en el embarazo, preparación para el parto, planeación para el recién nacido, atención del lactante y vida familiar adecuada, puede ser muy valiosa para ayudar a la adolescente. Los programas educacionales que ofrecen asistencia psicológica pueden fomentar también una conducta más responsable y madura. Los contactos de la adolescente con el personal profesional durante el embarazo pueden ser su única fuente de información en cuanto a sexualidad, enfermedades venéreas y anticoncepción.

Nuestra experiencia recomienda para lograr una mayor y mejor atención obstétrica en la adolescente, un trabajo de equipo, en el que deben participar: Padres, Médicos, Enfermeras, Psicólogos y Trabajadoras Sociales. Además se deben iniciar campañas tendientes a impartir una adecuada educación sexual (no tan sólo información) desde temprana edad en nuestra población.

\section{BIBLIOGRAFIA}

1. Acuña RH. Boletín de la Oficina Sanitaria Panamericana. Editorial El Ayudante Médico. Vol. LXXXII. 1977; 537-538.

2. Alborada L. La Adolescencia. Revista Mayo-Junio 1982; 22.

3. Allers R. Pedagogía Sexual. Barcelona. Editorial Luis Miracle 1981.

4. Angulo NA. Situación Social de Colombia.

5. Barrios AJ., Ramos OA. Aspectos Perinatales de las adolescentes. Clínica de Maternidad Rafael Calvo de Cartagena, 1983. Rev. Col. Obstet. Ginecol. 1985; 35: 182.

6. Berustein J. Diagnóstico y tratamiento de las relaciones familiares. Ediciones Horme, S.A.-E. Editorial Paidós, Buenos Aires 1980.

7. Briones G. La Formación de problemas de investigación social. Editorial Carrera 7a. Ediciones Uniandes 1981.

8. Córdoba R. Reflexiones acerca de la atención integral de la adolescente. Rev. Antio. Méd. Medellín 1974; 78-84.

9. Sapiña RA. Medicina de Adolescente. Clínicas Médicas de Norteamérica. Edit. Interamericana $1975 ; 512$.

10. Duarte CA., Barreto LA. Factores determinantes del embarazo en adolescentes solteras. Rev. Col. Obstet. Ginecol. 1985; 36(5): 291-306.

11. Erison E. Infancia y Sociedad. Ediciones Horme, 2a. Ed. Buenos Aires 1978; 235-237.

12. El Espectador. Embarazo en adolescentes problema creciente. 1981; Secc B. Pág. 1B.

13. Gutiérrez De PV. Estructura, función y cambio en Colombia. 1980.

14. Huffman.J. Ginecología en la infancia y en la adolescente. Editorial Salvat, Barcelona. 1971; 589-606.

15. Embarazos Juveniles, S.A. Imprenta Hispanoamericana. Mallorca, S. Barcelona, España. 1971; 592.
16. I.C.B.F. Carta de derecho de familia No. 17. 1984 Bogotá.

17. I.P.P.F. 1984 B. 18 No. 2. Pág. A. 1984.

18. Jeanniere A. Antropología Sexual. Editorial Luis Miracle, Barcelona, 1981.

19. Ladrón De GL. Metodología de la Investigación Científica. Edición 2a. Impreso U. Santo Tomás. 1984.

20. López IJJ. Libro de la Vida Sexual. Barcelona. Editorial Danac. 1979.

21. López PA. La familia una incógnita para la juventud. Ediciones Sol y Luna. 1977.

22. Mays O. La problemática del adolescente. Vol. 8, Edit. Dr. Vivente Constanzo. 1975; 11-115.

23. Marun G. Manual de investigación en sicología social. Editorial Trillas México. 1975.

24. Mora G. Formación de adolescentes. Editorial compañía continental. Edición México 1982; 320-325.

25. Revista del Jueves No. 38 El Espectador. Madres solteras desde los 14 años. 1982.

26. Rizo A., Prada E. La fecundidad de la población de 10 a 19 años en Colombia. Revista Colombiana de Obstetricia y Ginecología. Vol. XXXII No. 6 1982; 357-365.

27. Ucrós CA., Casas L., Ucrós S. El adolescente en Colombia. Revista Médica 1982; 5: 11-25.

28. Velasco FR. Los problemas del adolescente. Salud Pública de México. $1984 ; 529-562$.

29. Werdain H. Bomes. Clínicas Médicas de Norteamérica. Medicina de adolescentes. 1975; 1409. 